\title{
A!
}

This is an electronic reprint of the original article.

This reprint may differ from the original in pagination and typographic detail.

Latifi, Kourosh; Kopitca, Artur; Zhou, Quan

\section{Rapid mode-switching for acoustic manipulation}

\section{Published in:}

Proceedings of the 4th International Conference on Manipulation, Automation, and Robotics at Small Scales, MARSS 2019

DOI:

10.1109/MARSS.2019.8860952

Published: 01/07/2019

Document Version

Peer reviewed version

Please cite the original version:

Latifi, K., Kopitca, A., \& Zhou, Q. (2019). Rapid mode-switching for acoustic manipulation. In S. Haliyo, A. Sill, Q. Zhou, P. Kallio, \& S. Fatikow (Eds.), Proceedings of the 4th International Conference on Manipulation, Automation, and Robotics at Small Scales, MARSS 2019 [8860952] IEEE.

https://doi.org/10.1109/MARSS.2019.8860952

This material is protected by copyright and other intellectual property rights, and duplication or sale of all or part of any of the repository collections is not permitted, except that material may be duplicated by you for your research use or educational purposes in electronic or print form. You must obtain permission for any other use. Electronic or print copies may not be offered, whether for sale or otherwise to anyone who is not an authorised user. 
(C) 2019 IEEE. This is the author's version of an article that has been published by IEEE. Personal use of this material is permitted. Permission from IEEE must be obtained for all other uses, in any current or future media, including reprinting/republishing this material for advertising or promotional purposes, creating new collective works, for resale or redistribution to servers or lists, or reuse of any copyrighted component of this work in other works. 


\title{
Rapid mode-switching for acoustic manipulation
}

\author{
Kourosh Latifi ${ }^{1}$, Artur Kopitca ${ }^{1}$, and Quan Zhou ${ }^{1}$
}

\begin{abstract}
Acoustic manipulation techniques are becoming increasingly common with a range of applications in the biomedical research, microassembly, and lab-on-a-chip. Recently, a class of devices have attracted considerable attention which utilize dynamic and reconfigurable acoustic fields, known as dynamic-field acoustic devices. A common method of applying dynamic fields is to use mode-switching by rapidly altering the excitation frequency. Such methods generally rely on the switching being performed faster than the time constant associated with the particle motion. Nevertheless, it remains a grand challenge to eliminate or at least reduce the switching time to a minimal value. In this paper, we suggest employing a high-speed controller to minimize the switching time, enabling continuous particle manipulation in a dynamic-field acoustic device. As a proof-of-concept, we apply such idea to a classic acoustic manipulation device, a Chladni plate which consists of a centrally-actuated vibrating plate. By employing a closed-loop real-time controller, we show successful manipulation of particles on the plate on predefined trajectories. The high-speed switching methodology can also be applied to other dynamic-field acoustic methods, such as surface acoustic wave (SAW) devices, acoustic levitators, and in-fluid acoustic devices. This can result in faster and smoother particle manipulation in such devices.
\end{abstract}

Index Terms-Acoustic manipulation; real-time control; dynamic-filed acoustic device; mode-switching; Chladni plate; model predictive control

\section{INTRODUCTION}

The use of acoustic techniques in handling of miniature objects has seen a rapid development in recent years [1]. Acoustic techniques facilitate operations, such as particle manipulation [2], [3], pattern formation [4], and microassemly [5]. These techniques benefit numerous applications in the biological research [6], tissue engineering [7], and lab-on-a-chip [1].

Traditionally, acoustic manipulation devices rely on static patterns of acoustic pressure, commonly referred to as staticfield acoustic devices [1]. In such devices, a common configuration is to excite a resonance in a fluid-filled chamber or channel [2], [8]-[10]. Any specific device has an infinite number of possible resonant modes to choose from; however, typically a single low-order mode with a simple mode-shape is selected [1]. Hence the acoustic field is fixed by the resonant mode-shape, which is a characteristic of the geometry and acoustic properties of the device. Therefore, such

This work was supported by Academy of Finland (grant 296250), and Aalto doctoral school of Electrical Engineering

${ }^{1}$ Kourosh Latifi, Artur Kopitca, and Quan Zhou are with Department of Electrical Engineering and Automation, Aalto University, 02150 Espoo, Finland kourosh.latifidaalto.fi, artur.kopitcadaalto.fi, quan.zhoudaalto.fi devices only facilitate simple manipulation tasks, whereas more advanced manipulation tasks require more versatile and reconfigurable devices [1].

Recent years have also seen the emergence of devices which capitalize on dynamic and reconfigurable acoustic fields [1]. A dynamic-field acoustic device is one in which the acoustic field is altered dynamically for the purpose of particle manipulation. Dynamic acoustic fields lead to acoustic forces that change with time. They have opened up the possibility of performing a wide range of manipulations, such as the transportation and rotation of individual particles or agglomerates [11], [12]. In such devices, multiple methods have been suggested to alter the acoustic field with time.

A method of introducing dynamic fields is to use modeswitching in which the acoustic field within the device is rapidly switched between several resonant modes [1], [13]. In such devices, the current position of the particle is measured, and the appropriate mode is then selected and played to direct the particle towards a target. This approach has been applied in SAW devices [6] and in-plane manipulators [14].

The mode-switching acoustic methods generally rely on the switching being performed faster than the time constant associated with the particle motion [1]. In other words, the switching time must be sufficiently low to prevent any notable transient effects on particle motion. Although much research has been devoted to exploit such devices, it remains a grand challenge to eliminate or at least reduce the switching time to a minimal value.

In this paper, we suggest employing a high-speed controller to minimize the switching time. We apply such idea to a centrally-actuated vibrating plate, commonly referred to as a Chladni plate, which is a classic acoustic manipulation device [3], [15]. In our device, the high-speed controller calculates the best mode to play, and switches the frequency accordingly. By changing the driving frequency, the plate offers complex frequency-dependent two-dimensional excitation fields on the plate surface. Those fields allow us to control the motion of sub-mm objects on predefined trajectories.

Additionally, we experimentally show that the modeswitching is performed faster than the time constant associated with the particle motion in our device. Here the highspeed controller enables rapid mode-switching with a short delay of approximately 2 milliseconds, almost two orders of magnitude faster than our previous implementation [3], [15].

This paper is organized as follows. In Section II, the experimental setup is introduced. In Section III, we explain the high-speed control methodology which includes the experimental procedure to model the motion of particles, the 

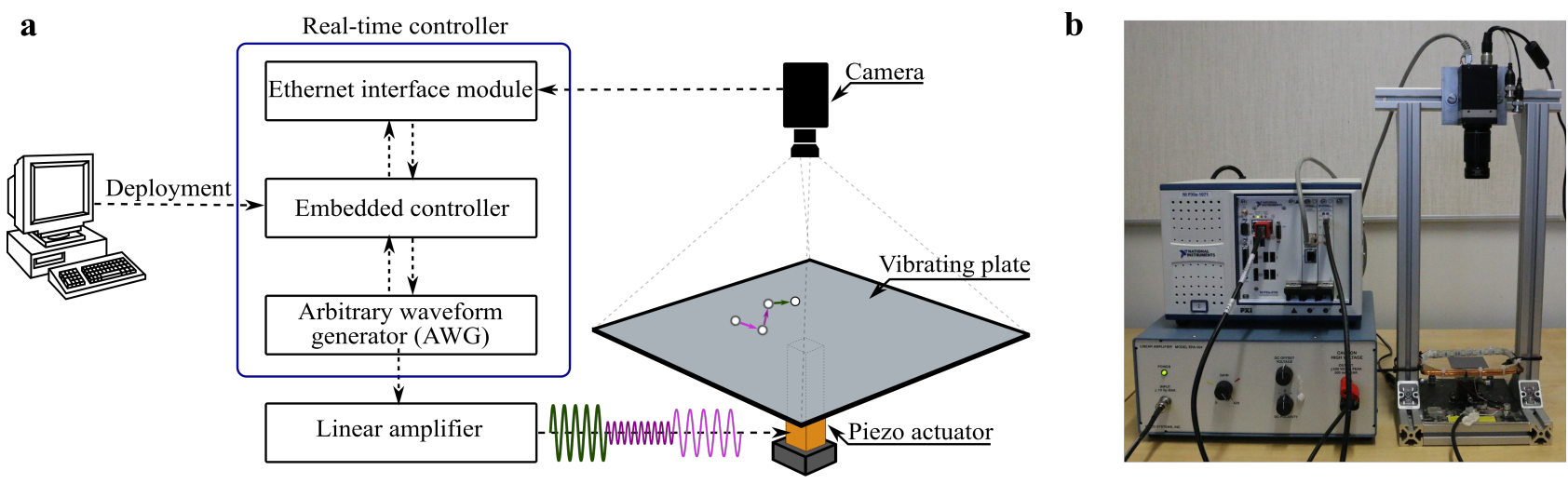

Fig. 1. (a) Schematic of the experimental device, consisting of a vibrating plate mounted on a piezoelectric actuator. Motion of the particle is recorded by a camera, and based on the current position of the particle, a real-time controller calculates a control signal that moves the particle towards the target position. (b) Experimental setup.

control software architecture, the applied control algorithm, and the signal generation method. The experimental results to evaluate our control approach are presented in Section IV. Finally, conclusions and perspectives of this work are discussed in Section V.

\section{APPARATUS}

The apparatus consists of a centrally-actuated vibrating plate, as shown in Fig. 1. The vibrating plate has dimensions of $50 \mathrm{~mm} \times 50 \mathrm{~mm} \times 50 \mu \mathrm{m}$, diced from a silicon wafer and glued on a piezoelectric actuator (Piezomechanik, PSt $150 / 2 \times 3 / 20$ ) using cyanoacrylate adhesive. The piezoelectric actuator is mounted on a dual-axis goniometer (Thorlabs, GN2/M). The longitudinal vibration of the piezoelectric actuator, which is oriented perpendicular to the plate, excites the bending modes of the plate. We use pressed solder balls (Martin Smt/VD90.5106, Sb96.5Ag3Cu0.5, $\varnothing 600 \mu \mathrm{m})$ as manipulation specimens. The particle and plate are imaged from above by a video camera (ImperX, IGV-B1621C-KC000 with Infinity/InfiniMite Alpha lens). The camera is connected to an embedded controller (National Instruments, PXIe-8135) via an Ethernet interface module (National Instruments, PXIe-8234) to feedback the position of the particle. To provide better contrast, a strip of flexible LEDs (NEXTEC, LS5300NWIP20) is mounted horizontally around and slightly above the plate. The plate is excited with sinusoidal signals at a frequency of $1-30 \mathrm{kHz}$. The frequency of the signal is calculated in the embedded controller. The signal is then generated in an arbitrary waveform generator (AWG) (National Instruments, PXI-5412), amplified by a linear amplifier (Piezo Systems, EPA-104-230), and sent to the piezoelectric actuator. The embedded controller, the Ethernet interface module, and the AWG are mounted on a Chassis (National Instruments, PXIe-1071).

\section{HiGH-SPEED CONTROL METHODOLOGY}

In this section, we explain the main elements of our high-speed control methodology, including the procedure for modelling the particle motion on the Chladni plate, the control software architecture, the implemented closed-loop control scheme, and the signal generation method.

\section{A. Modelling of the motion}

To model the particle motion on the plate, we apply particle tracking velocimetry [3] to build models that can predict the motion of particles at a given location and frequency. We evenly distribute $600 \mu \mathrm{m}$ pressed solder balls on the plate, and play the notes in the Chromatic musical scale from 1 to $30 \mathrm{kHz}$ (59 distinct frequencies). The notes are triangularly-enveloped sinusoidal signals with a nominal amplitude calculated similar to [3]. The notes are played in a random order, and each note repeated 25 times. The position $\mathbf{P}$ of each particle and displacement $\Delta \mathbf{P}$ after the note has been played are measured by the camera. For each note, we create a data set $(\mathbf{P}, \Delta \mathbf{P})$, which includes $x$ and $y$ components of the position and displacement. The plate is then divided into a $21 \times 21$ grid and all data points within one grid cell are averaged. For each note, we fit a model $\mathbf{d}_{n}$ to $(\mathbf{P}, \Delta \mathbf{P})$ dataset by performing robust LOESS regression (locally weighted scatterplot smoothing) on the binned values (span $7.5 \mathrm{~mm}$ ) as follows,

$$
(\Delta x, \Delta y)=\mathbf{d}_{n}(x, y)+\mathbf{r}_{n}(x, y) .
$$

To calculate the uncertainty of the model, we compute the error for each data point as follows,

$$
|e|^{2}=\left|(\Delta x, \Delta y)-\mathbf{d}_{n}(x, y)\right| \text {. }
$$

We then form a dataset $\left(\mathbf{P}, e^{2}\right)$, and use similar fitting procedure to compute $\mathbf{r}_{n}$ for each note. We then calculate $\mathbf{d}_{n}$ and $\mathbf{r}_{n}$ for the corresponding frequencies at evenly-spaced $21 \times 21$ grids over the plate coordinates, and create a database of the models. The database is stored on the hard drive of the embedded controller. We apply bilinear interpolation to calculate the expected displacement at a query point inside a grid. 


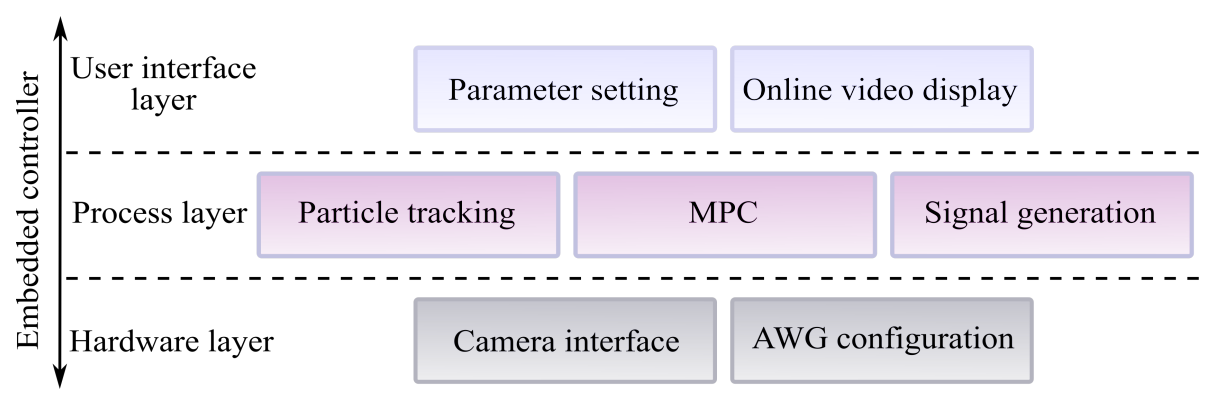

Fig. 2. Three-layer software architecture of the Real-time controller.

\section{B. Control software architecture}

We have implemented a three-layer software architecture within the embedded controller using NI LabVIEW system design software, as shown in Fig. 2. The architecture includes a hardware layer, a process layer, and a user interface layer. The LabVIEW platform includes built-in functionality for communicating data between each layer, and also displaying it on a PC.

The hardware layer handles the low-level driver functions required to operate the apparatus. The components in this layer include the camera interface and the AWG configuration blocks. The camera interface captures the camera recording via NI Vision Acquisition Software at a frame rate of approximately $42 \mathrm{fps}$ and a quality of 8-bit grayscale. Every frame is then sent to the process layer where the particle detection is performed. The AWG configuration block produces precise sine waveforms of the manipulation frequency.

The process layer provides the algorithms for the manipulation system by using LabVIEW 2017 Real-Time Module. This layer processes the video images, detects the particle, and makes control decisions based on the model database. To detect the particle, we use the blob detection algorithm of NI Vision Development Module. The corresponding parameters are tuned in the User interface layer. The detected position is then fed to a closed-loop controller explained in Section IIIC.

The user interface layer includes a menu for setting the parameters of manipulation. This includes the image adjustment and the particle detection parameters, such as the brightness and the approximate size of the particle. It also displays live image data from the camera, the controller state, the signal generation state, and the $x$ and $y$ coordinates of the particle on a host PC.

\section{Motion control}

We have implemented a closed-loop control scheme to plan and control the motion of a particle on the plate [15]. We use a camera to capture the top-view of the plate, and repeatedly measure the position of the objects on the plate. In every step, we use the model to choose a note that moves the particle towards the desired direction. For such purpose, we use model predictive control (MPC). Our model predictive controller is based on iterative optimization of the nonlinear cost function $J$ for a finite horizon. At time step $t$, position of the object is sampled and the optimization cost function $J$ is calculated for all mode combinations until time horizon $T$. The optimization cost function $J$ is represented as,

$$
J_{n}=\sum_{i=1}^{T}\left|\mathbf{S}-\mathbf{E}\left(x_{i}, y_{i}\right)\right|,
$$

where $i$ denotes the current time horizon, $\mathbf{S}$ the target position of the particle, and $\mathbf{E}\left(x_{n}, y_{n}\right)$ the expected position of the object in time horizon $i$ as a function of the particle current position $\left(x_{n}, y_{n}\right)$. In time horizon 1 , we use the measured current location of the particle instead of expected position. In every time step, only the first calculated note is played, then the position is sampled again and the calculations are repeated starting from the new current position. We used $T=1$ (greedy controller) in our control experiments presented in Section IV.

\section{Signal generation}

In our manipulation experiments explained in Section IV, we use sinusoidal signals (Fig. 3) of the following form,

$$
s(t)=D C+k \times g_{n} \times \sin \left(2 \pi f_{n} t\right),
$$

where $s$ is the time-varying excitation signal, $D C$ the bias voltage, $k$ the manipulation gain, $g_{n}$ the nominal amplitude of the note $n$, and $f_{n}$ the frequency of the note $n$.

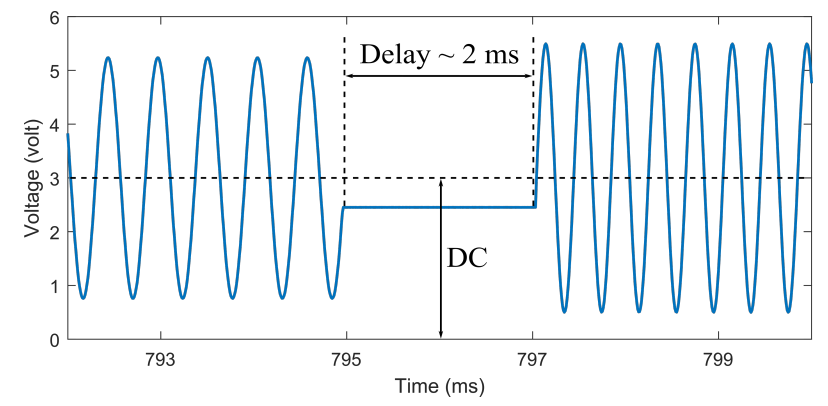

Fig. 3. Excitation signal and the switching delay.

We use $D C=3$ and $k=4.1$ as the bias voltage and the manipulation gain, respectively. In our control experiments 

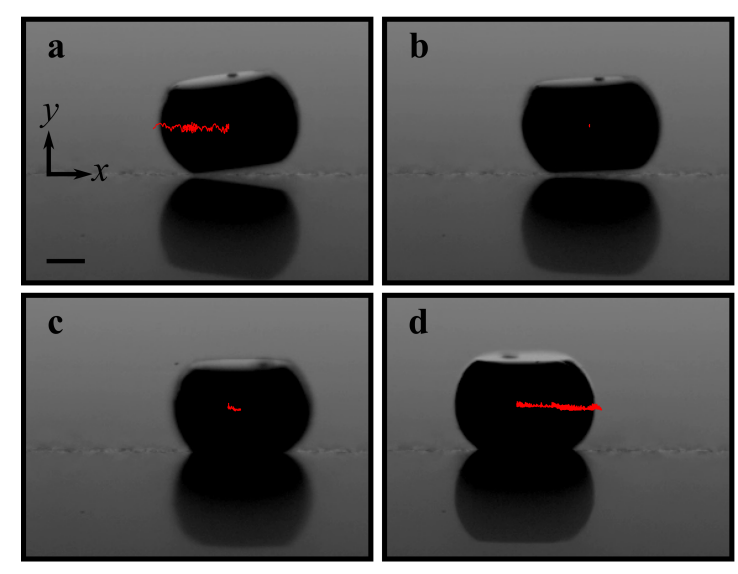

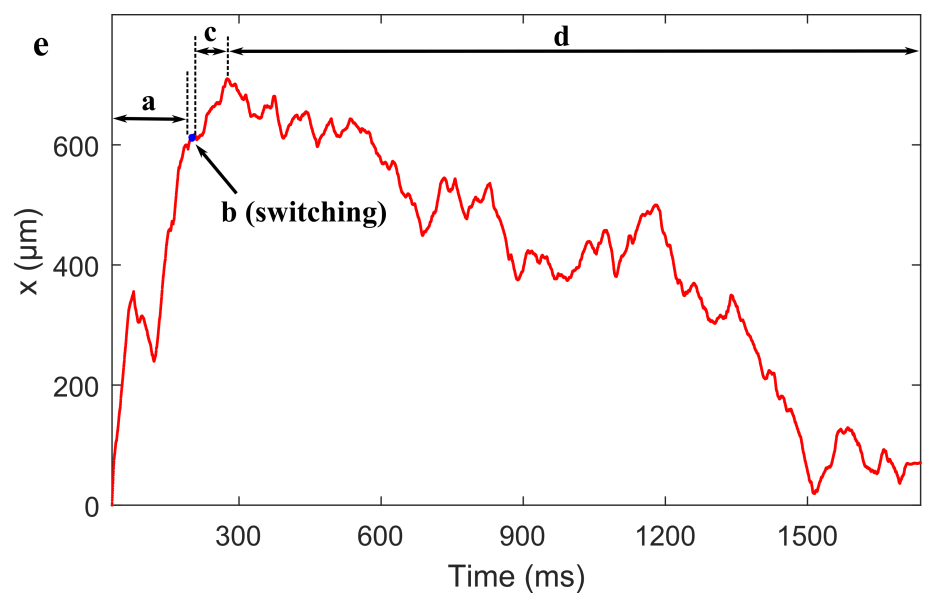

Fig. 4. Motion of a $600 \mu \mathrm{m}$ pressed solder ball on the Chladni plate: (a) The plate is excited with a frequency of $6271.93 \mathrm{~Hz}$ (the note $G_{8}$ ) for 168 milliseconds, and the particle moves to the right edge of the plate. For (a-d) the red dots show the trails of the motion. (b) The excitation is paused due to mode-switching for around 2 milliseconds, while the particle is still moving. (c) The particle still moves by its inertia towards the direction of the first note for nearly 73 milliseconds, even though the second note is played. (d) The particle moves back towards the center point by the second excited note (the note $G_{7}^{\#}$ ). (e) Particle lateral position vs. time. Scale bar, $300 \mu \mathrm{m}$.

presented in Section IV, we amplify the signal $s$ via a voltage amplifier by a voltage gain of 20 .

To generate the signal $s$, we use National Instruments PXI-5412 which is a $20 \mathrm{MHz}$ AWG capable of generating user-defined arbitrary waveforms as well as standard functions, such as sine, square, triangle, and ramp. We prefer the AWG solution to a simple Function Generator (FG) or an Arbitrary Function Generator (AFG), since it is capable of generating sinusoidal signals with an arbitrary voltage bias in a broad range of frequencies. We generate the signals in the Arbitrary Waveform output mode of the AWG. This AWG uses direct digital synthesis (DDS) to precisely generate waveforms. The AWG uses an onboard memory to store the sine waveforms constructed of $N$ datapoints with a nominal amplitude of $g_{n}$. After each control step, the frequency of the selected note $f_{n}$ is used to calculate the signal sample rate $F_{n}$ as below,

$$
F_{n}=N \times f_{n} .
$$

We use $N=256$ in this work. The signal sample rate is then sent to the AWG to generate the corresponding signal. When the controller decides which frequency to play, the signal generation initiates until the new control command is received. If the new control command still includes the previous frequency, the AWG continues to generate the same signal without stopping. However, if the controller commands to switch the frequency, the signal generator switches the signal with a relatively short delay. According to our measurements, the delay is in the range of approximately 2 milliseconds, as shown in Fig. 3 .

\section{RESUlts}

\section{A. Effect of mode-switching on the particle motion}

We have experimentally investigated the effect of modeswitching on the particle motion, as shown in Fig. 4. To perform such an experiment, we place a pressed solder ball on the surface of the plate at the center point. A high-speed video camera (Phantom v2012) with a macro lens (Canon MP-E 65mm f/2.8 1-5X Macro Photo) is installed on a tripod at one side of the apparatus to observe the particle-plate interaction with a frame rate of $5000 \mathrm{fps}$. We select two notes which have displacement field parallel to the image plane of the high-speed camera at the center point of the plate. We then excite the plate with a frequency of $6271.93 \mathrm{~Hz}$ (the note $G_{8}$ ) for 168 milliseconds, which moves the particle laterally towards the right edge of the plate, as shown in Fig. 4a. Then we switch the frequency to $3322.44 \mathrm{~Hz}$ (the note $G_{7}^{\#}$ ) for 1500 milliseconds. While switching the note, the excitation of the plate is paused for approximately 2 milliseconds, as discussed in Section III-D. Figure $4 \mathrm{~b}$ shows the motion of the particle while the excitation signal is paused due to mode-switching. The mode-switching does not stop the particle motion and the particle still moves towards the same direction by its inertia (Fig. 4c). After a while, roughly 73 milliseconds in our experiment, the particle moves back towards the center point by the second excited note (the note $G_{7}^{\#}$ ), as shown in Fig. 4d. After stopping the excitation, the particle continues its motion for approximately 39 milliseconds. Figure $4 \mathrm{e}$ shows the lateral position of the particle during the experiment. This experiment shows that the mode-switching is performed considerably faster than the time constant of the plate-particle interaction in our system.

\section{B. Control experiments}

We have successfully demonstrated motion control of particles using the real-time controller, as shown in Fig. 5. We have manipulated a $600 \mu \mathrm{m}$ solder ball on different trajectories, including a $\mathrm{z}$-shaped, rectangle-shaped, and triangle-shaped trajectories in approximately 69, 114, and 115 seconds, respectively. To control the motion of the 
a

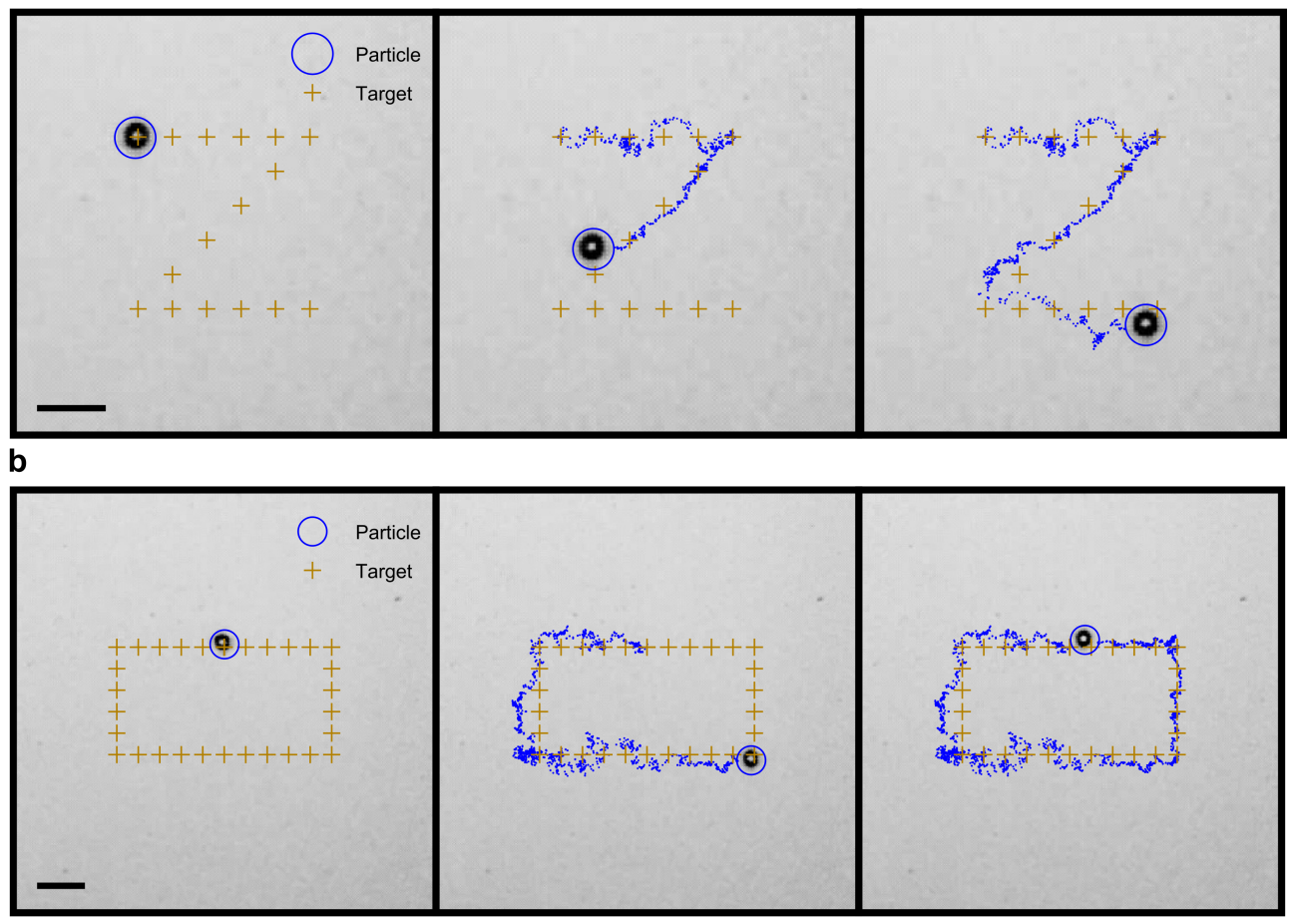

C

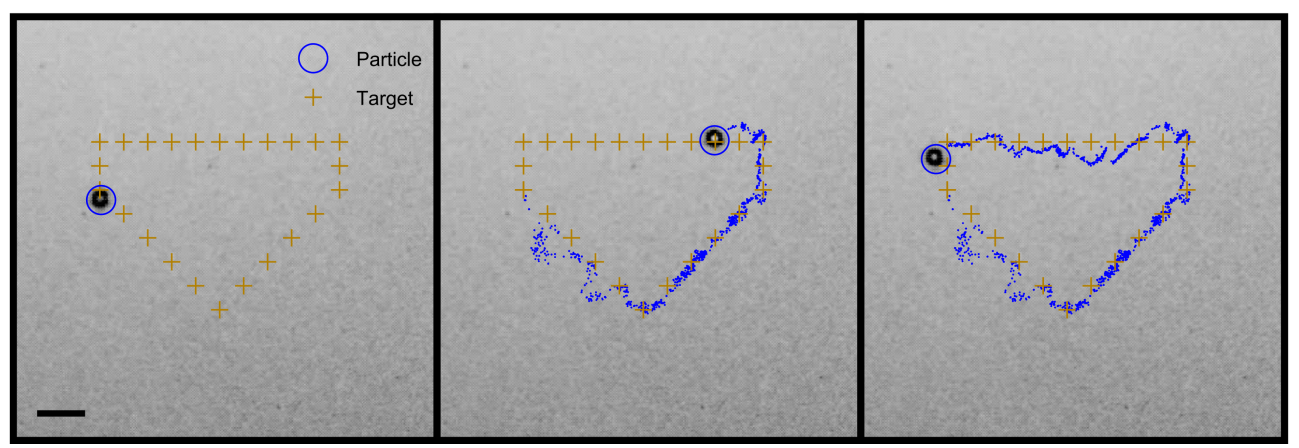

Fig. 5. Manipulation of a $600 \mu \mathrm{m}$ pressed solder ball on a (a) Z-shaped, (b) rectangle-shaped, and (c) triangle-shaped trajectories. Scale bar, 2 mm.

particle, we split the trajectory into a set of waypoints and store them on the hard drive of the embedded controller. During the manipulation experiments, the embedded controller reads the sequence of the defined waypoints. We use a sequence of notes to direct the particle to its current waypoint. Once a frame is captured by the camera, the current position of the particle is measured. The measured position is then fed to the MPC controller. The controller searches in the database of the modelled notes, and selects the note that directs the particle towards the current waypoint. The selected note is played on the vibrating plate until the next image frame is received by the controller. The controller excites the plate until the distance between the particle and its waypoint is less than a predefined threshold. Then the algorithm directs the particle towards the next waypoint along the trajectory.

\section{Concurrency for high-speed control}

In our previous works [3], [15], the closed-loop controller performed multiple computational processes in series including the image processing, particle detection, control optimization, and signal generation. This resulted in a relatively long computational process of approximately 500 milliseconds, allowing merely pulse-based excitation of the system. Unlike our previous works, here we apply two concurrent computational processes in the closed-loop control scheme. The first process involves online video processing and control optimization which is performed at the order of microseconds for each control step. Therefore, the control process is considerably faster than the frame rate of the camera which captures one frame in approximately every 23 milliseconds. The second process includes the signal generation that is performed continuously. If the excitation frequency changes, the mode-switching is performed with a short delay of approximately 2 milliseconds, as shown in Fig. 3. As a result, our approach 
in the present work, using the concurrent computational processes by a real-time controller, facilitates continuous manipulation of the particle. This shows that the concurrency of the computational processes by a high-speed controller greatly reduces the pausing time of manipulation by over two orders of magnitude.

\section{Conclusions}

This paper has presented a new methodology for continuous manipulation in dynamic-field acoustic methods. The fundamental idea is to employ a high-speed controller which reduces the switching time between two acoustic fields to a minimal value. We implemented such idea to a classic example of an acoustic manipulation device, a Chladni plate, and showed that the switching time can be reduced to approximately 2 milliseconds, which is considerably below the associated time constant of motion in such device. We have successfully shown controlled manipulation of sub-mm objects on predefined trajectories on the plate. The method is applicable to other types of particles with different shapes and materials, similar to the ones reported in [3], [15]. The high-speed switching methodology can also be applied to other dynamic-field acoustic methods, such as SAW devices, acoustic levitators, and in-fluid acoustic devices. This can result in faster and smoother particle manipulation by means of dynamic-field acoustic methods.

\section{ACKNOWLEDGMENT}

The authors acknowledge Micronova Nanofabrication Centre for providing laboratory facilities for microfabrication, and Harri Wijaya for his comments during the course of this work.

\section{REFERENCES}

[1] B. W. Drinkwater, "Dynamic-field devices for the ultrasonic manipulation of microparticles," Lab Chip, vol. 16, no. 13, pp. 2360-2375, 2016.

[2] T. Laurell, F. Petersson, and A. Nilsson, "Chip integrated strategies for acoustic separation and manipulation of cells and particles," Chem. Soc. Rev., vol. 36, no. 3, pp. 492-506, 2007.

[3] Q. Zhou, V. Sariola, K. Latifi, and V. Liimatainen, "Controlling the motion of multiple objects on a Chladni plate," Nat. Commun., vol. 7, pp. 1-10, 2016.

[4] G. Vuillermet, P. Y. Gires, F. Casset, and C. Poulain, "Chladni Patterns in a Liquid at Microscale," Phys. Rev. Lett., vol. 116, no. 18, pp. 184501, 2016.

[5] J. Goldowsky, M. Mastrangeli, L. Jacot-Descombes, M. R. Gullo, G. Mermoud, J. Brugger, A. Martinoli, B. J. Nelson, and H. F. Knapp, "Acousto-fluidic system assisting in-liquid self-assembly of microcomponents," J. Micromechanics Microengineering, vol. 23, no. 12, pp. 125026, 2013.

[6] X. Ding, S.-C. S. Lin, B. Kiraly, H. Yue, S. Li, I.-K. Chiang, J. Shi, S. J. Benkovic, and T. J. Huang, "On-chip manipulation of single microparticles, cells, and organisms using surface acoustic waves," Proc. Natl. Acad. Sci., vol. 109, no. 28, pp. 11105-11109, 2012.

[7] S. Li, P. Glynne-Jones, O. G. Andriotis, K. Y. Ching, U. S. Jonnalagadda, R. O. C. Oreffo, M. Hill, and R. S. Tare, "Application of an acoustofluidic perfusion bioreactor for cartilage tissue engineering," Lab Chip, vol. 14, no. 23, pp. 4475-4485, 2014.
[8] M. Wiklund, "Acoustofluidics 12: Biocompatibility and cell viability in microfluidic acoustic resonators," Lab Chip, vol. 12, no. 11, p. 2018-2028, 2012.

[9] J. Shi, X. Mao, D. Ahmed, A. Colletti, and T. J. Huang, "Focusing microparticles in a microfluidic channel with standing surface acoustic waves (SSAW)," Lab Chip, vol. 8, no. 2, pp. 221-223, 2008.

[10] Z. Wang and J. Zhe, "Recent advances in particle and droplet manipulation for lab-on-a-chip devices based on surface acoustic waves," Lab Chip, vol. 11, no. 7, p. 1280-1285, 2011.

[11] D. Ahmed, A. Ozcelik, N. Bojanala, N. Nama, A. Upadhyay, Y. Chen W. Hanna-Rose, and T. J. Huang, "Rotational manipulation of single cells and organisms using acoustic waves," Nat. Commun., vol. 7, pp. 11085, 2016.

[12] K. Melde, E. Choi, Z. Wu, S. Palagi, T. Qiu, and P. Fischer, "Acoustic Fabrication via the Assembly and Fusion of Particles," Adv. Mater. vol. 30, no. 3, pp. $1704507,2018$.

[13] P. Glynne-Jones, R. J. Boltryk, N. R. Harris, A. W. J. Cranny, and M. Hill, "Mode-switching: A new technique for electronically varying the agglomeration position in an acoustic particle manipulator," Ultrasonics, vol. 50, no. 1, pp. 68-75, 2010.

[14] A. Haake and J. Dual, "Contactless micromanipulation of small particles by an ultrasound field excited by a vibrating body," J. Acoust. Soc. Am., vol. 117, no. 5, pp. 2752-2760, 2005.

[15] K. Latifi, H. Wijaya, and Q. Zhou, "Multi-particle acoustic manipulation on a Chladni plate," in International Conference on Manipulation, Automation and Robotics at Small Scales (MARSS), 2017. 